\title{
Examination of surgical and conservative treatment effects on depression of patients with moderate carpal tunnel syndrome
}

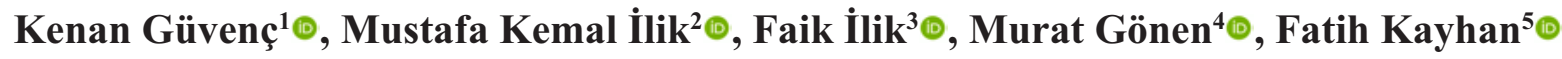 \\ ${ }^{1}$ Department of Orthopedics, Mersin University School of Medicine, Mersin, Turkey \\ ${ }^{2}$ Department of Neurosurgery, Farabi Hospital, Konya, Turkey \\ ${ }^{3}$ Department of Neurology, Konya Medicana Hospital, Konya, Turkey \\ ${ }^{4}$ Department of Neurology, Firat University School of Medicine, Elazı̆̆, Turkey \\ ${ }^{5}$ Consultant Psychiatrist, Soul Psychiatry Private Practice, Konya, Turkey
}

DOI: $10.18621 /$ eurj.414363

\begin{abstract}
Objective: The aim of this study is to examine the outcomes of conservative and surgical treatments of patients with carpal tunnel syndrome as well as the effects of these two treatment options on depressive symptoms of these patients.

Methods: A case-control study carried out from June 2015 to Fabruary 2016 and 111 moderate moderate carpal tunnel syndrome patients were included in the study whose diagnosis was confirmed by electroneuromyography (ENMG). The 78 of the patients had surgical treatment and 33 of them, who did not accept the surgery, received steroids via injection and splinting technique was performed for 8 weeks. Boston Carpal Tunnel Questionnaire, quick disabilities of the arm, shoulder and hand score and Beck Depression Invertory scales were applied six months before and after treatments.

Results: It was detected that pain functional assessment scores and Beck depression scores significantly recovered the disease in patients who had surgical treatment compared to ones who had the conservative treatment.

Conclusions: It can be concluded that surgical treatment plays a more active role in the recovery of depressive symptoms in patients with moderate carpal tunnel syndrome.
\end{abstract}

Keywords: Carpal tunnel syndrome, surgical, conservative, depression

Received: April 11, 2018; Accepted: July 9, 2018; Published Online: September 27, 2018

$\mathrm{C}$ arpal tunnel syndrome (CTS) occurs due to the compression of the median nerve in the wrist alignment $[1,2]$. Its prevalence is $2.7 \%$ [2, 3]. It can lead to paresthesia, pain, weakness and sometimes thenar atrophy in the median nerve innervation area [4]. The physiological basis of CTS depends on the median nerve ischemia as a result of the increased pressure in the carpal tunnel due to the transverse carpal ligament compression or circulatory disorders
[5]. Besides, tenosynovitis of the tendons nearby nerve is the other hypothesis that tries to explain the CTS. Collagen vascular diseases, metabolic diseases, pregnancy and wrist fractures or dislocations can lead to CTS [6]. However, it is substantially idiopathic. It can be diagnosed with the help of anamnesis, physical examination and electrophysiological tests [7]. Decompressive surgery and local corticosteroid injections are the recommended treatment options. According to pre-

Address for correspondence: Kenan Güvenç, MD., Mersin University School of Medicine, Department of Orthopedics, Mersin, Turkey E-mail: guvenckenan@hotmail.com, Fax: +903243610073 
vious studies, neurophysiological parameters improved in patients who had the surgical treatment [8]. It was been observed that neuropathic pain lead to depression in CTS patients [9]. In CTS, the relation between depressive symptoms and the outcome of surgery is of particular interest because depression and CTS are both highly prevalent conditions, specifically among women $[10,11]$. There is no published evidence about the optimal treatment method for mild disease [12]. Some studies have reported no effect of depression on the outcome of treatment of CTS, others stated that depression affects scar pain or patient satisfaction and perceived disability [13-15]. The aim of this study is to compare the effects of surgical and conservative treatments on the depression levels of patients with moderate CTS.

\section{METHODS}

A case-control study carried out from June 2015 to Fabruary 2016 and 111 moderate CTS patients were included in the study whose diagnosis was confirmed by electroneuromyography (ENMG). The CTS classification was performed by nerve conduction studies. The findings were assessed according to the Padua criteria and CTS classification was performed. The electrophysiological classification criteria of Padua are:

1) Most severe: It is the 'most severe' when motor and sensory action potential cannot be obtained.

2) Severe: It is 'severe' when there is no sensory response and there is an abnormal motor distal response.

3) Moderate: It is 'moderate' when there are sensory conduction and motor abnormalities together in the finger wrist segment.

4) Mild: It is 'mild' when there are abnormalities in the sensory conduction speed in the finger wrist segment and when the distal motor latency is normal.

5) Light: It is 'light' when there are abnormalities in the finger wrist segment according to the comparative assessment.

\section{6) Normal}

The 3rd group of Padua (moderate CTS patients) was included in our study since there is no consensus on the treatment of moderate carpal tunnel syndrome in the literature. Out of 111 patients, 78 of them had the surgical treatment and 33 of them, who did not accept the surgery, had the conservative treatment. Surgical treatment was performed by using standard mini-open incision technique. All operations were conducted under the local anesthesia. Transverse carpal ligament was reached with the help of one mini incision (approximately $2-3 \mathrm{~cm}$ ) on the third long web axis. An attempt was made to protect median and ulnar nerves in the palmar cutaneous branch during the dissection. Carpal tunnel was loosened from antebrachial fascia till transverse carpal ligament along the ulnar edge. Steroid injection and 8 weeks splinting treatments were performed in the conservative conservative treatment. Boston Carpal Tunnel Syndrome Questionnaire BCTSQ, Beck Depression Inventory (BDI), and Quick Disability of the Arm, Shoulder, and Hand (qDASH) surveys were performed before and 6 months after the treatment. BCTSQ is a disease specific survey. It is composed of the two scales which evaluate the severity of symptoms and the functional capacity. There is a Turkish validity and reliability study of the scale and some studies suggest that it is useful to use it in the effectiveness of the treatment $[16,17]$. There are five different responses which can be scored between 1 and 5. The mean score is obtained by dividing the total score to the total number of questions and it changes between 1 and 5. High score indicates that the functional capacity decreases. The mean score is calculated separately for the symptom severity and functional capacity. Symptom score is composed of 11 and function score is composed of 8 questions.

Out of 11 titles, at least 10 titles should be answered in order to calculate qDASH score. Each title contains five answers and the score of the scale is calculated by using title scores (0: no disability, 100: most severe disability) $[18,19]$. BDI test was used in order to determine the presence of the depressive signs and severity of these symptoms. BDI is a scale which is composed of 21 questions for the evaluation of the depression signs classified into three areas such as physical, emotional, cognitive. The maximum score can be 63. According to BDI scores, scores between 10 and 16 represent mild, scores between 17 and 29 represent moderate and scores between 30 and 63 represent severe depressive symptoms. The cut-off point of the scale is 17 and score higher than 17 represents significant clinical depressive signs [20]. 
Table1. Demographic features of the patients

\begin{tabular}{lccc}
\hline & Surgical group & Conservative group & $\boldsymbol{p}$ value \\
\hline Age, mean (range) (years) & $53.5(21-77)$ & $51(20-81)$ & 0.14 \\
Sex & & & 0.07 \\
$\quad$ Male (n) & 71 & 6 & \\
Female (n) & & 27 & \\
\hline
\end{tabular}

Patients' ages and hand dominance were recorded. The exclusion criteria of the study were; being in the period of pregnancy or lactation, having an anamnesis of wrist fracture or dislocation, being diagnosed with polyneuropathy and previously diagnosed with a psychiatric disease.

\section{Statistical analysis}

Results were statistically compared to each other by using SPSS program. Kolmogrov Smirnov test was used to assess whether or not the variables were normally distributed. Chi-square or Fischer exact tests (when needed) were used in order to compare the categorical variables. Mann Whitney U test was used to analyze the numerical variables.

\section{RESULTS}

The 111 moderate CTS patients were included in the study. The 97 of the patients were female and 14 of the patients were male. The dominant hand was the right hand in 104 of the patients whereas the dominant hand was left hand in 9 of the patients. The mean age of the patients was 52.7 years. The surgical treatment was applied to 78 patients and conservative treatment was applied to 33 patients. There was no difference between these two groups in terms of their demographic characteristics (Table 1).

There was a statistically significant recovery in patients who were treated with surgical intervention compared to ones who received conservative treatment according to their BCTSQ, qDASH and BDI scores (Table 2).

\section{DISCUSSION}

Even though CTS is the most frequently observed peripheral nerve entrapment syndrome, there are problems in its diagnosis and treatment $[1,2,21]$. Although the endoscopic technique has been commonly used, the gold standard in CTS surgery is the open carpal tunnel release. Steroid injections, splinting, exercise, low level laser therapy, ultrasound therapy and paraffin bath can be applied in the conservative treatment of CTS $[22,23]$. It has been already shown in previous studies that there is an association between the pain intensity and the depression [9]. It has also been specified that neuropathic pain can lead to depression and anxiety with the effect of nociceptive responses in animal

Table 2. Outcomes of rating scales

\begin{tabular}{lcccc}
\hline Rating scale & \multicolumn{2}{c}{ Surgical group } & \multicolumn{2}{c}{ Conservative group } \\
\cline { 2 - 5 } & Pre-treatment & Post-treatment & Pre-treatment & Post-treatment \\
\hline BCTQ & $4.08 \pm 0.15$ & $1.20 \pm 0.11^{*}$ & $4.09 \pm 0.15$ & $2.28 \pm 0.35^{*}$ \\
FS & $3.77 \pm 0.15$ & $1.89 \pm 1.96^{*}$ & $3.78 \pm 0.13$ & $2.43 \pm 0.30^{*}$ \\
SS & $24.07 \pm 2.51$ & $6.21 \pm 2.76^{*}$ & $24.81 \pm 2.51$ & $17.87 \pm 2.64^{*}$ \\
BDS & $35.95 \pm 8.14$ & $4.72 \pm 2.97^{*}$ & $36.08 \pm 8.34$ & $25.96 \pm 3.53^{*}$ \\
qDASH score & Data are given as mean \pm standard deviation. BCTQ = Boston carpal tunnel questionnaire, FS = functional status, \\
SS = symptoms severity, BDS = Beck depression scale, qDASH = quick disabilities of the arm, shoulder and \\
hand score, $* p<0.01$
\end{tabular}


models [24]. It has been thought that chronic pain leads to depression since the peripheral inflammation reaches the central nervous system. It has also been considered in animal models that tumor necrosis factor- $\alpha(\mathrm{TNF}-\alpha)$ can be the key mediator which explains the association of depression and the neuropathic pain [25].

In a previous study, patients who were operated had better results compared to the ones who had the steroid injections in terms of symptomatic and neurophysiological outcomes [26]. When these two treatment methods were compared to each other, it was shown in another study that the results of patients who had surgical treatment were better in the end of the two years compared to others [27]. Similar to other studies, it was shown in our study that surgically treated patients had prominent recovery according to the patients who were treated conservatively. In our study, another important finding was that the depression frequency and the severity of patients who were treated with the surgical method were lower compared to the other patients treated with conservative methods. In a previous study, it was shown that the emotional status of CTS patients was evaluated and it was shown that depression was correlated with the diminished sensation of hand, hand weakness, thenar atrophy and pain $[28,29]$. It was considered that the recovery in the depressive signs of patients who received surgical treatment was due to the prominent decrease in the pain and functional healing.

\section{CONCLUSION}

Conclusively, surgical treatment is superior to the conservative treatment in the moderate CTS according to pain and function hand scorings. As a result of these findings, surgical treatment should be prioritized in moderate CTS patients.

\section{Conflict of interest}

The authors disclosed no conflict of interest during the preparation or publication of this manuscript.

\section{Financing}

The authors disclosed that they did not receive any grant during conduction or writing of this study.

\section{REFERENCES}

[1] Andreu JL, Ly-Pen D, Millán I, de Blas G, Sánchez-Olaso A. Local injection versus surgery in carpal tunnel syndrome: neurophysiologic outcomes of a randomized clinical trial. Clin Neurophysiol 2014;125:1479-84.

[2] Bakhtiary AH, Rashidy-Pour A. Ultrasound and laser therapy in the treatment of carpal tunnel syndrome. Aust J Physiother 2004;50:147-51.

[3] Baptista-de-Souza D, Nunciato AC, Pereira BC, Fachinni G, Zaniboni CR, Canto-de-Souza A. Mice undergoing neuropathic pain induce anxiogenic-like effects and hypernociception in cagemates. Behav Pharmacol 2015;26:664-72.

[4] Baysal O, Altay Z, Ozcan C, Ertem K, Yologlu S, Kayhan A. Comparison of three conservative treatment protocols in carpal tunnel syndrome. Int J Clin Pract 2006;60:820-8.

[5] Beaton DE, Wright JG, Katz JN. Development of the QuickDASH: comparison of three item-reduction approaches. J Bone Joint Surg Am 2005;87:1038-46.

[6] Bonfiglioli R, Mattioli S, Fiorentini C, Graziosi F, Curti S, Violante FS. Relationship between repetitive work and the prevalence of carpal tunnel syndrome in part-time and full-time female supermarket cashiers: a quasi-experimental study. Int Arch Occup Environ Health 2007;80:248-53.

[7] Burt S, Crombie K, Jin Y, Wurzelbacher S, Ramsey J, Deddens J. Workplace and individual risk factors for carpal tunnel syndrome. Occup Environ Med 2011;68:928-33.

[8] Cobb TK, Amadio PC, Leatherwood DF, Schleck CD, Ilstrup DM. Outcome of reoperation for carpal tunnel syndrome. J Hand Surg Am 1996;21:347-56.

[9] de Krom MC, de Krom CJ, Spaans F. [Carpal tunnel syndrome: diagnosis, treatment, prevention and its relevance to dentistry]. Ned Tijdschr Tandheelkd 2009;116:97-101. [Article in Dutch]

[10] Datema M, Tannemaat MR, Hoitsma E, van Zwet EW, Smits F, van Dijk JG, et al. Outcome of carpal tunnel release and the relation with depression. J Hand Surg Am 2018;43:16-23.

[11] Shin YH, Yoon JO, Kim YK, Kim JK. Psychological status is associated with symptom severity in patients with carpal tunnel syndrome. J Hand Surg Am. 2018;43: 484.e1-484.e8.

[12] Ebrahimzadeh MH, Mashhadinejad H, Moradi A, Kachooei AR. Carpal tunnel release in diabetic and non-diabetic patients. Arch Bone Jt Surg 2013;1:23-7.

[13] Hobby JL, Venkatesh R, Motkur P. The effect of psychological disturbance on symptoms, self-reported disability and surgical outcome in carpal tunnel syndrome. J Bone Joint Surg Br 2005;87:196-200.

[14] Becker SJ, Makanji HS, Ring D. Expected and actual improvement of symptoms with carpal tunnel release. J Hand Surg Am 2012;37:1324-9.e1-5.

[15] Kim JK, Kim YK. Predictors of scar pain after open carpal tunnel release. J Hand Surg Am 2011;36:1042-6.

[16] Fernández-de-las-Peñas C, Fernández-Muñoz JJ, PalaciosCeña M, Navarro-Pardo E, Ambite-Quesada S, Salom-Moreno J. Direct and indirect effects of function in associated variables such as depression and severity on pain intensity in women with carpal tunnel syndrome. Pain Med 2015;16:2405-11. 
[17] Heybeli N, Ozerdemoglu RA, Aksoy OG, Mumcu EF. [Functional and symptomatic scoring used for the assessment of outcome in carpal tunnel release]. Acta Orthop Traumatol Turc 2001; 35:147-51. [Article in Turkish]

[18] Hisli N. A study about validity of Beck depression inventory. J Psychol 1988;6:118-22.

[19] Hui AC, Wong S, Leung CH, Tong P, Mok V, Poon D, et al. A randomized controlled trial of surgery vs steroid injection for carpal tunnel syndrome. Neurology 2005;64:2074-8.

[20] Koldas Dogan S, Ay S, Evcik D, Baser O. Adaptation of Turkish version of the questionnaire Quick Disability of the Arm, Shoulder, and Hand (Quick DASH) in patients with carpal tunnel syndrome. Clin Rheumatol 2011;30:185-91.

[21] Ly-Pen D, Andréu JL, Millán I, de Blas G, Sánchez-Olaso A. Comparison of surgical decompression and local steroid injection in the treatment of carpal tunnel syndrome: 2-year clinical results from a randomized trial. Rheumatology (Oxford) 2012;51:1447-54.

[22] Papanicolaou GD, McCabe SJ, Firrell J. The prevalence and characteristics of nerve compression symptoms in the general population. J Hand Surg Am 2001;26:460-6.

[23] Pogorzelski R, Kułakowska A, Halicka D, Drozdowski W.
[Neurological and emotional profile of carpal tunnel syndrome patients]. Przegl Lek. 2011;68:269-73. [Article in Polish] [24] Robert A, Werner R, Andray M. Electrodiagnostic evaluation of carpal tunnel syndrome. Muscle Nerve 2011;44:597-607.

[25] Salaffi F, De Angelis R, Grassi W; MArche Pain Prevalence; Investigation Group (MAPPING) study. Prevalence of Musculoskeletal conditions in an Italian population sample: results of a regional community-based study. I. The MAPPING study. Clin Exp Rheumatol 2005;23:819-28.

[26] Sen D, Chhaya S, Morris VH. Carpal tunnel syndrome. Hosp Med 2002;63:392-5.

]27] Verdugo RJ, Salinas RS, Castillo J, Cea JG. Surgical versus non-surgical treatment for carpal tunnel syndrome. Cochrane Database Syst Rev. 2003;(3):CD001552. Review. Update in: Cochrane Database Syst Rev 2008;(4):CD001552.

[28] Yücel H, Seyithanoğlu H. Choosing the most efficacious scoring method for carpal tunnel syndrome. Acta Orthop Traumatol Turc 2015;49:23-9.

[29] Lozano Calderon SA, Paiva A, Ring D. Patient satisfaction after open carpal tunnel release correlates with depression. J Hand Surg Am 2008;33:303-7. 